\title{
Evolution equations for light-cone distribution amplitudes of heavy-light hadrons
}

\author{
V. M. Braun \\ Institut für Theoretische Physik, Universität Regensburg \\ D-93040 Regensburg, Germany \\ E-mail: vladimir.braundur.de
}

QCD factorization approach for heavy hadron decays introduces light-cone distribution amplitudes for heavy mesons and baryons as the main nonperturbative input. Evolution equations for these distributions have interesting properties under conformal transformations and are completely integrable for several important cases which allows one to obtain explicit solutions. The limit of large light quark energies in higher-twist distributions appears to be nontrivial. In this region the hierarchy of contributions with rising anomalous dimensions is lost and their resummation is mandatory. 


\section{Introduction}

B-meson light-cone distribution amplitudes (DAs) are the main nonperturbative input to the QCD description of weak decays involving light hadrons in the final state [1,2,3]. In particular the leading-twist DA $\phi_{+}(\omega)$ gives a dominant contribution in the heavy quark expansion and it received considerable attention already $[4,5,6,7]$. In particular an explicit solution for the corresponding renormalization group (evolution) equation has been found $[8,9,10]$. It turns out that such equations have nontrivial mathematical properties under collinear conformal transformation of the light-cone coordinates [9]. This symmetry explains the structure of the solution and, in fact, allows one to find it without knowing the explicit expression for the evolution kernel. More symmetries are uncovered going over to three-particle DAs (with either a light quark and a gluon, or two light quarks) which turn out to be completely integrable, i.e. they have a nontrivial integral of motion. This property allows one to find explicit solutions of the evolution equations for threeparticle DAs [11, 12] and also find the scale dependence of the subleading twist two-particle DA $\phi_{-}(\omega)$ in the limit of large number of colors $[11,12]$, which proves to be remarkably simple: it is the same as for the leading-twist DA $\phi_{+}(\omega)$ up to a constant shift in the anomalous dimension [12].

These recent results offer a consistent framework for the construction of B-meson and $\Lambda_{b}$ baryon DAs as an expansion in contributions that have autonomous scale dependence and a minimum amount of nonperturbative parameters. This is important since utility of the QCD factorization techniques depends on the possibility to control, or at least estimate, the corrections suppressed by powers of the $b$-quark mass that involve higher-twist DAs. This task is attracting increasing attention and in the last years there have been several efforts to combine light-cone sum rules (LCSRs) with the expansion in terms of B-meson DAs $[13,14,15,16]$. The LCSR technique allows one to tame infrared divergences which appear power-suppressed contributions in the purely perturbative framework and to calculate the so-called soft or end-point nonfactorizable contributions in terms of the DAs of increasing twist. One of the problems on this way is that higher-twist Bmeson DAs involve contributions of multiparton states that are very poorly known. Our results are a step in this direction.

\section{Conformal symmetry of the Lange-Neubert evolution equation}

Following an established convention we define the B-meson DA as the renormalized matrix element of the bilocal operator built of an effective heavy quark field $h_{v}(0)$ and a light antiquark $\bar{q}(z n)$ at a light-like separation:

$$
\left\langle 0\left|\bar{q}(z n) \not h[z n, 0] \Gamma h_{v}(0)\right| \bar{B}(v)\right\rangle=-\frac{i}{2} F(\mu) \operatorname{Tr}\left[\gamma_{5} \not h \Gamma P_{+}\right] \Phi_{+}(z, \mu) .
$$

Here $[z n, 0]$ is the light-like Wilson line operator, $v_{\mu}$ is the heavy quark velocity, $n_{\mu}$ is the lightlike vector, $n^{2}=0$, such that $n \cdot v=1, P_{+}=\frac{1}{2}(1+\not \hat{~})$ is the projector on upper components of the heavy quark spinor, $\Gamma$ stands for an arbitrary Dirac structure, $|\bar{B}(v)\rangle$ is the $\bar{B}$-meson state in the heavy quark effective theory (HQET) and $F(\mu)$ is the decay constant in HQET, which is used for normalization. 
The invariant function $\Phi_{+}(z, \mu)$ where $z$ is a real number defines what is usually called the leading twist B-meson DA in position space. Its Fourier transform is

$$
\phi_{+}(\omega, \mu)=\frac{1}{2 \pi} \int_{-\infty}^{\infty} d z e^{i \omega z} \Phi_{+}(z-i 0, \mu), \quad \Phi_{+}(z, \mu)=\int_{0}^{\infty} d \omega e^{-i \omega z} \phi_{+}(\omega, \mu),
$$

where in the first equation the integration contour goes below the singularities of $\Phi_{+}(z, \mu)$ that are located in the upper-half plane. The parameter $\mu$ is the renormalization (factorization) scale. We tacitly imply using dimensional regularization with modified minimum subtraction.

The scale dependence of the DA is driven by the renormalization of the corresponding nonlocal operator

$$
O_{+}(z)=\bar{q}(z n) \not l[z n, 0] \Gamma h_{v}(0) .
$$

The corresponding one-loop Z-factor was computed by Lange and Neubert (LN) [5], giving rise to an evolution equation which is convenient to write, for our purposes, as a renormalization group equation for the operator $O_{+}(z)[6,17]$ :

$$
\left(\mu \frac{\partial}{\partial \mu}+\beta(g) \frac{\partial}{\partial g}+\frac{\alpha_{s}}{2 \pi} H_{L N}\right) O_{+}(z, \mu)=0,
$$

where

$$
\left[H_{L N} f\right](z)=2 C_{F}\left[\int_{0}^{1} \frac{d \alpha}{\alpha}(f(z)-\bar{\alpha} f(\bar{\alpha} z))+\ln (i \mu z) f(z)-\frac{5}{4} f(z)\right], \quad \bar{\alpha} \equiv 1-\alpha .
$$

The main result of Ref. [11] is that the LN kernel can be written in terms of the generator of special conformal transformations

$$
H_{L N}=2 C_{F}\left[\ln \left(i \mu S^{+}\right)-\psi(1)-\frac{5}{4}\right]
$$

where

$$
S_{+}=z^{2} \partial_{z}+2 j z, \quad S_{0}=z \partial_{z}+j, \quad S_{-}=-\partial_{z},
$$

satisfy standard $S L(2)$ commutation relations

$$
\left[S_{+}, S_{-}\right]=2 S_{0}, \quad\left[S_{0}, S_{ \pm}\right]= \pm S_{ \pm} .
$$

Going over to momentum space corresponds to using a different (adjoint) representation for the generators:

$$
\widetilde{S}_{+}=i\left[\omega \partial_{\omega}^{2}+2 j \partial_{\omega}\right], \quad j=1 .
$$

Note that the scale $\mu$ under the logarithm in (2.5) is necessary simply because $S_{+}$has dimension $[\text { mass }]^{-1}$.

This result can be derived in several ways, e.g., starting from the commutation relations for the LN kernel obtained in Ref. [17]:

$$
\left[S_{+}, \mathscr{H}_{L N}\right]=0, \quad\left[S_{0}, \mathscr{H}_{L N}\right]=2 C_{F} .
$$


Since the problem has one degree of freedom - the light-cone coordinate of the light quark it follows from $\left[S_{+}, \mathscr{H}\right]=0$ that the operator $\mathscr{H}$ must be a function of $S_{+}, \mathscr{H}=h\left(S_{+}\right)$. This function can be found using the second commutation relation. Let $S=S_{0}+1$. Then $S_{+}=z S$ and the relation $\left[S_{0}, h\left(S_{+}\right)\right]=2 C_{F}$ can be written equivalently as $[S, h(z S)]=2 C_{F}$. Taking into account that $[S, z S]=z S$ one obtains an equation on the function $h(s)$

$$
s h^{\prime}(s)=2 C_{F} \quad \Longrightarrow \quad h(s)=2 C_{F}[\ln s+\text { constant }],
$$

reproducing the expression in Eq. (2.5) up to a (scheme-dependent) integration constant.

The main advantage of Eq. (2.5) is that diagonalization of the LN kernel $H_{L N}$ can be traded for a much simpler task of finding the eigenfunctions of the first-order differential operator $S_{+}$(2.6):

$$
i S_{+} Q_{s}(z)=s Q_{s}(z) \quad \Rightarrow \quad Q_{s}(z)=-\frac{1}{z^{2}} \exp \left\{\frac{i s}{z}\right\} .
$$

The anomalous dimensions are found easily by evaluating

$$
H_{L N} Q_{s}(z)=2 C_{F}\left[\ln \left(i \mu S^{+}\right)-\psi(1)-\frac{5}{4}\right] Q_{s}(z)=2 C_{F}\left[\ln (\mu s)-\psi(1)-\frac{5}{4}\right] Q_{s}(z) .
$$

Going over to the momentum space $Q_{s}(z)$ are replaced by Bessel functions [11] and the final result for the evolution of the leading-twist B-meson DA reads

$$
\begin{aligned}
\phi_{+}(\omega, \mu) & =\int_{0}^{\infty} d s \sqrt{\omega s} J_{1}(2 \sqrt{\omega s}) \eta_{+}(s, \mu) \\
\eta_{+}(s, \mu) & =R\left(s ; \mu, \mu_{0}\right) \eta_{+}\left(s, \mu_{0}\right)
\end{aligned}
$$

where

$$
R\left(s ; \mu, \mu_{0}\right)=\exp \left[-\int_{\mu_{0}}^{\mu} \frac{d \tau}{\tau} \Gamma_{\text {cusp }}\left(\alpha_{s}(\tau)\right) \ln \left(\frac{\tau s}{s_{0}}\right)\right]=\left(\frac{\mu}{\mu_{0}}\right)^{-\frac{2 C_{F}}{\beta_{0}}}\left(\frac{\mu_{0} s}{s_{0}}\right)^{\frac{2 C_{F}}{\beta_{0}} \ln L} L^{-\frac{4 C_{F} \pi}{\beta_{0}^{2} \alpha_{s}\left(\mu_{0}\right)}}
$$

with $s_{0}=e^{5 / 4-\gamma_{E}}$ and $L=\alpha_{s}(\mu) / \alpha_{s}\left(\mu_{0}\right)$.

The same result was obtained independently in Ref. [8]. In their notation $s \eta(s, \mu) \mapsto \rho_{+}(1 / s, \mu)$.

\section{Twist-three distribution amplitudes}

To the twist-three accuracy two more DAs enter the game,

$$
\begin{aligned}
& \left\langle 0\left|\bar{q}(n z) \hbar \hbar \gamma_{5} h_{v}(0)\right| \bar{B}(v)\right\rangle=i F(\mu) \Phi_{-}(z, \mu), \\
& \left\langle 0\left|\bar{q}\left(n z_{1}\right) g G_{\mu v}\left(n z_{2}\right) n^{v} \sigma^{\mu \rho} n_{\rho} \gamma_{5} h_{v}(0)\right| \bar{B}(v)\right\rangle=-2 i F(\mu) \Phi_{3}\left(z_{1}, z_{2}, \mu\right),
\end{aligned}
$$

where $\Phi_{3}=\Psi_{A}-\Psi_{V}$ in the commonly accepted notation [18]. These DAs are related to each other by the QCD equation of motion (EOM) [3, 18]

$$
\left(1+z \partial_{z}\right) \Phi_{-}(z, \mu)=\Phi_{+}(z, \mu)+2 \int_{0}^{z} w d w \Phi_{3}(z, w, \mu)
$$


that can be solved to obtain $\Phi_{-}(z, \mu)$ as a sum of the so-called Wandzura-Wilczek (WW) term expressed in terms of $\Phi_{-}(z, \mu)$ [3], and a certain integral of the quark-gluon DA $\Phi_{3}\left(z_{1}, z_{2}, \mu\right)$. The latter contribution is nontrivial as it involves a function of two variables. We find, however [12], that this complication is to a large extent illusory as the particular integral appearing in the EOM, $\int_{0}^{z} w d w \Phi_{3}(z, w, \mu)$, evolves autonomously in the large $N_{c}$ limit.

Evolution equation for $\Phi_{3}\left(z_{1}, z_{2}, \mu\right)$ takes the form

$$
\left(\mu \frac{\partial}{\partial \mu}+\beta(g) \frac{\partial}{\partial g}+\frac{\alpha_{s}}{2 \pi} \mathscr{H}\right) F(\mu) \Phi_{3}\left(z_{1}, z_{2}, \mu\right)=0
$$

where the "Hamiltonian" $\mathscr{H}$ to the one-loop accuracy is given by a sum of two-particle kernels

$$
\mathscr{H}=H_{q g}+H_{g h}+H_{q h} .
$$

For our present purposes it is convenient to write the kernels in terms of the generators of $\operatorname{SL}(2)$ transformations $[19,9]$

$$
\begin{aligned}
& H_{q g}=N_{c}\left[\psi\left(J_{q g}+3 / 2\right)+\psi\left(J_{q g}-3 / 2\right)-2 \psi(1)-3 / 4\right]+\frac{2}{N_{c}}(-1)^{J_{q g}-3 / 2} \frac{\Gamma\left(J_{q g}-3 / 2\right)}{\Gamma\left(J_{q g}+3 / 2\right)}, \\
& H_{g h}=N_{c}\left[\ln \left(i \mu S_{g}^{+}\right)-\psi(1)-1 / 2\right], \quad H_{q h}=-\frac{1}{N_{c}}\left[\ln \left(i \mu S_{q}^{+}\right)-\psi(1)-5 / 4\right],
\end{aligned}
$$

where $H_{q h}$ is the heavy-light quark LN kernel (with a different color factor), $H_{g h}$ is the gluon-heavy quark kernel that involves the generator $S_{g}^{+}$with conformal spin $j=3 / 2$, and $J_{q g}$ is defined in terms of the corresponding quadratic Casimir operator $J_{q g}\left(J_{q g}-1\right)=\left(\vec{S}_{q}+\vec{S}_{g}\right)^{2}$.

It turns out that the leading contribution to $\mathscr{H}$ for a large number of colors

$$
\mathscr{H}=N_{c} \mathbb{H}+N_{c}^{-1} \delta \mathbb{H},
$$

possesses a nontrivial "hidden" symmetry. Namely, it is possible to construct two "conserved charges", $\mathbb{Q}_{1}$ and $\mathbb{Q}_{2}$, that commute between themselves and with the large- $N_{c}$ evolution kernel $\mathbb{H}$ :

$$
\left[\mathbb{Q}_{1}, \mathbb{Q}_{2}\right]=\left[\mathbb{Q}_{1}, \mathbb{H}\right]=\left[\mathbb{Q}_{2}, \mathbb{H}\right]=0
$$

where

$$
\mathbb{Q}_{1}=i\left(S_{q}^{+}+S_{g}^{+}\right), \quad \mathbb{Q}_{2}=\frac{9}{4} i S_{g}^{+}-i S_{g}^{+}\left(S_{g}^{+} S_{q}^{-}+S_{g}^{0} S_{q}^{0}\right)-i S_{g}^{0}\left(S_{q}^{0} S_{g}^{+}-S_{g}^{0} S_{q}^{+}\right) .
$$

The "conserved charges" $\mathbb{Q}_{1}, \mathbb{Q}_{2}$ and the "Hamiltonian" $\mathbb{H}$ are self-adjoint operators with respect to the $S L(2)$ scalar product [12]. It follows that they have real eigenvalues and can be diagonalized simultaneously. Their common eigenfunctions $Y_{s, x}\left(z_{1}, z_{2}\right)$ can be found using the method developed in Ref. [20]:

$$
Y_{s, x}\left(z_{1}, z_{2}\right)=\frac{i s^{2}}{z_{1}^{2} z_{2}^{3}} \int_{0}^{1} d u u \bar{u} e^{i s\left(u / z_{1}+\bar{u} / z_{2}\right)}{ }_{2} F_{1}\left(\begin{array}{c}
-\frac{1}{2}-i x,-\frac{1}{2}+i x \\
2
\end{array} \mid-\frac{u}{\bar{u}}\right),
$$

where $s>0$ and $-\infty<x<\infty$, and a special solution

$$
Y_{s}^{(0)}\left(z_{1}, z_{2}\right)=\frac{i s^{2}}{z_{1}^{2} z_{2}^{3}} \int_{0}^{1} d u u \bar{u} e^{i s\left(u / z_{1}+\bar{u} / z_{2}\right)}
$$


that corresponds to the imaginary value of the second parameter, $Y_{s}^{(0)}\left(z_{1}, z_{2}\right)=Y_{s, x=i / 2}\left(z_{1}, z_{2}\right)$. The corresponding eigenvalues of $N_{c} \mathbb{H}$ are

$$
\begin{aligned}
& \gamma_{s, x}=N_{c}[\ln (\mu s)+\psi(3 / 2+i x)+\psi(3 / 2-i x)-3 \psi(1)-5 / 4], \\
& \gamma_{0} \equiv \gamma_{s, x=i / 2}=N_{c}[\ln (\mu s)-\psi(1)-1 / 4],
\end{aligned}
$$

so that one obtains a continuum spectrum of anomalous dimensions and a discrete level separated from the continuum by a finite gap $\Delta \gamma=\gamma_{s, 0}-\gamma_{0}=N_{c}[2 \psi(3 / 2)-\psi(2)-\psi(1)]=0.227411 N_{c}$.

A generic three-particle DA $\Phi_{3}\left(z_{1}, z_{2}, \mu\right)$ can be expanded in the eigenfunctions of the large- $N_{c}$ evolution kernel

$$
\Phi_{3}\left(z_{1}, z_{2}, \mu\right)=\int_{0}^{\infty} d s\left[\eta_{0}(s, \mu) Y_{s}^{(0)}\left(z_{1}, z_{2}\right)+\frac{1}{2} \int_{-\infty}^{\infty} d x \eta(s, x, \mu) Y_{s, x}\left(z_{1}, z_{2}\right)\right],
$$

where the coefficient functions $\eta_{0}(s, \mu)$ and $\eta(s, x, \mu)$ have autonomous scale dependence up to $1 / N_{c}^{2}$ corrections:

$$
\begin{gathered}
\eta_{0}(s, \mu)=L^{N_{c} / \beta_{0}} R\left(s ; \mu, \mu_{0}\right) \eta_{0}\left(s, \mu_{0}\right), \\
\eta(s, x, \mu)=L^{\gamma_{x} / \beta_{0}} R\left(s ; \mu, \mu_{0}\right) \eta\left(s, x, \mu_{0}\right),
\end{gathered}
$$

where $R\left(s ; \mu, \mu_{0}\right)$ is defined in (2.15) and

$$
\gamma_{x}=N_{c}\left[\psi(3 / 2+i x)+\psi(3 / 2-i x)+2 \gamma_{E}\right]
$$

The scale dependence of the two-particle twist-three DA can now be recovered from the EOM identity (3.2). Remarkably, all terms involving the hypergeometric function vanish thanks to the identity

$$
\int_{0}^{1} u d u_{2} F_{1}\left(\begin{array}{c}
-\frac{1}{2}-i x,-\frac{1}{2}+i x \\
2
\end{array} \mid-\frac{u}{\bar{u}}\right)=0,
$$

so that only the ground state (with the lowest anomalous dimension) contributes:

$$
\int_{0}^{z} w d w \Phi_{3}(z, w, \mu)=-\frac{1}{2 z^{2}} \int_{0}^{\infty} s d s e^{i s / z} \eta_{0}(s, \mu)
$$

Going over to the momentum space one obtains

$$
\phi_{-}(\omega, \mu)=\int_{0}^{\infty} d s\left[\eta_{+}(s, \mu)+\eta_{0}(s, \mu)\right] J_{0}(2 \sqrt{\omega s}) .
$$

The scale-dependence of the coefficient $\eta_{0}(s, \mu)$ differs from the leading-twist result for $\eta_{+}(s, \mu)$, Eq. (2.14), by a simple overall factor

$$
\eta_{0}(s, \mu)=L^{\Delta / \beta_{0}} R\left(s ; \mu, \mu_{0}\right) \eta_{0}\left(s, \mu_{0}\right), \quad \Delta=N_{c}+\mathscr{O}\left(1 / N_{c}\right) .
$$

In other words, the subleading twist contribution to $\phi_{-}(\omega, \mu)$ is suppressed at large scales as compared to the WW contribution by the universal factor $L^{\Delta / \beta_{0}}$ that does not depend on the light quark momentum. To the $\mathscr{O}\left(1 / N_{c}^{2}\right)$ accuracy there is no mixing with "genuine" quark-gluon degrees of freedom. 
It is tempting to define the "asymptotic" quark-gluon DA $\Phi_{3}^{\mathrm{as}}\left(z_{1}, z_{2}, \mu\right)$ as the contribution with the lowest anomalous dimension (for a given $s$ ):

$$
\Phi_{3}^{\mathrm{as}}\left(z_{1}, z_{2}, \mu\right)=\int_{0}^{\infty} d s \eta_{0}(s, \mu) Y_{s}^{(0)}\left(z_{1}, z_{2}\right) .
$$

The corresponding explicit expression in momentum space can be found in [12]. It has the expected behavior at small quark and gluon momenta, $\phi_{3}^{\text {as }}\left(\omega_{1}, \omega_{2}, \mu\right) \stackrel{\omega_{1}, \omega_{2} \rightarrow 0}{\sim} \mathscr{O}\left(\omega_{1} \omega_{2}^{2}\right)$, but, surprisingly, does not decrease for large gluon (and finite quark) momenta $\omega_{2} \rightarrow \infty$ which is seen as a power $1 / z_{2}$ singularity in coordinate space (gluon falling onto the Coulomb center). Contributions with higher anomalous dimensions from the continuum spectrum are even more singular, $\sim\left(1 / z_{2}\right)^{3 / 2 \pm i x}$, so that the corresponding momentum space contributions are increasing (and oscillating) functions of the gluon momentum.

We are able to show that all such singularities are, however, spurious and cancel in the sum of contributions of the asymptotic DA and the corrections. Most importantly, this cancellation is not spoiled by the evolution: The $\sim 1 / z_{2}$ singularity is not generated at higher scales provided it is not present already in the nonperturbative ansatz at a reference low scale. This result implies that for small $z_{2}$, alias large $\omega_{2} \gtrsim \mu$, the hierarchy of contributions with increasing anomalous dimensions is lost; the leading large- $\omega_{2}$ asymptotics of the "asymptotic" DA is exactly cancelled by the contributions with larger anomalous dimensions.

The way to see this is the following. The representation for $\Phi_{3}\left(z_{1}, z_{2}, \mu\right)$ (or equally its momentum space counterpart $\left.\phi_{3}\left(\omega_{1}, \omega_{2}, \mu\right)\right)$ contains an integral over real $x$-values and an additional contribution that can be thought of as coming from the point $x=i / 2$ in the complex plane. One can show [12] that the integration contour in $x$ can be moved into the complex plane $x$ to $x+i C$ with $1 / 2<C<3 / 2$ such that the special contribution is included, see figure, after which the behavior of
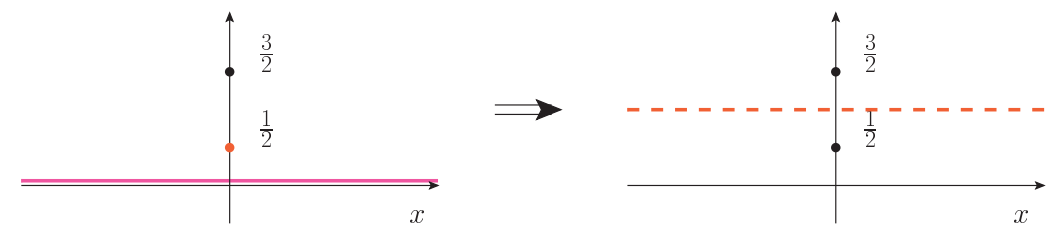

Figure 1: Contour deformation in the representation of the quark-gluon twist-three DA in multiplicatively renormalizable contributions.

the DA at small distances (large momenta) is determined by the position of the closest singularity of the integrand on the imaginary above the integration contour.

Taking into account the scale dependence amounts to the insertion of the RG factors (3.13) under the integral. In this way additional singularities appear corresponding to the poles of the anomalous dimension $\gamma_{x}$ (3.14). The singularity closest to the origin is at $x=(3 i) / 2$ so that if the initial condition for the evolution $\Phi_{3}\left(z_{1}, z_{2}, \mu_{0}\right)$ corresponds to a constant behavior at $z_{2} \rightarrow 0$, it will be modified to $\Phi_{3}\left(z_{1}, z_{2} \rightarrow 0\right) \sim \ln \left(\mu z_{2}\right)$, corresponding to a "tail" $1 / \omega_{2}$ in momentum space. The same behavior was found previously for the leading twist DA $[5,6,7]$. It is easy to see that in the other limit $\Phi_{3}\left(z_{1} \rightarrow 0, z_{2}\right) \sim \ln \left(\mu z_{1}\right)$ as well, so that our final conclusion is that gluon emission generates a radiative tail $\sim 1 / \omega_{1}$ and/or $\sim 1 / \omega_{2}$ of the three-particle DA $\phi_{3}\left(\omega_{1}, \omega_{2}, \mu\right)$ for both, large light quark and large gluon momenta. This is natural as the corresponding terms are present 
in the evolution kernels. The reason and consequences of such a behavior have been discussed at length in the literature, e.g. [6].

\section{Distribution amplitudes of heavy-light baryons}

The attention in flavor physics has so far mostly been focused on the meson sector. B-baryons are also produced copiously at $\mathrm{LHC}$, and have the advantage over the B-mesons of providing access to spin correlations in various baryon-to-baryon transitions. Especially semileptonic $\Lambda_{b}$ and $\Omega_{b}$ decays and their description in the QCD factorization framework are attracting increasing attention.

Although the DAs of the existing heavy baryons are all different, their scale dependence is similar. In particular the overall spin of the light quark pair ("diquark") is irrelevant and only the relative helicity of the two valence light quarks matters, so that the evolution is the same for the $j^{P}=0^{+} S U(3)_{F}$ triplet and all longitudinal DAs of heavy baryons in the $j^{P}=1^{+}$sextets, see [21]. The $\Lambda_{b}$ baryon is a prime example with light quarks having opposite helicity. Its DA is defined as $[22,21]$

$$
\left\langle 0\left|\left[u^{T}\left(z_{1} n\right) C \gamma_{5} \not h d\left(z_{2} n\right)\right] h_{v}(0)\right| \Lambda(v)\right\rangle=\Psi_{\Lambda}\left(z_{1}, z_{2} ; \mu\right) u_{\Lambda}(v) .
$$

For the baryons involving a spin-one diquark there are two possibilities, with aligned and antialigned helicities, and another DA

$$
\left\langle 0\left|\left[q_{1}^{T}\left(z_{1} n\right) C \not h \gamma_{\perp}^{\mu} q_{2}\left(z_{2} n\right)\right] h_{v}(0)\right| B^{j=1}(v)\right\rangle=\frac{1}{\sqrt{3}} \varepsilon_{\perp}^{\mu} u(v) \Psi_{\perp}\left(z_{1}, z_{2} ; \mu\right) .
$$

where $\varepsilon^{\mu}$ is the diquark polarization vector, $v^{\mu} \varepsilon_{\mu}=0$. The evolution equation has the standard form

$$
\left(\mu \frac{\partial}{\partial \mu}+\beta\left(\alpha_{s}\right) \frac{\partial}{\partial \alpha_{s}}+\frac{2 \alpha_{s}}{3 \pi} \mathbb{H}\right) f(\mu) \Psi\left(z_{1}, z_{2} ; \mu\right)=0
$$

The evolution kernel is an integral operators which, similar to the B-meson case, is a sum of twoparticle terms and can be written in terms of the generators of conformal transformations

$$
\begin{aligned}
& \mathbb{H}_{\uparrow \uparrow}=\ln \left(i \mu S_{+}^{(1)}\right)+\ln \left(i \mu S_{+}^{(2)}\right)+2 \psi\left(J_{12}\right)-4 \psi(2), \\
& \mathbb{H}_{\uparrow \downarrow}=\ln \left(i \mu S_{+}^{(1)}\right)+\ln \left(i \mu S_{+}^{(2)}\right)+2 \psi\left(J_{12}\right)-4 \psi(2)-\frac{1}{J_{12}\left(J_{12}-1\right)}
\end{aligned}
$$

for aligned and anti-aligned quark helicities, respectively. For the first case, which corresponds to transverse DAs of $j^{P}=1^{+}$sextets $\left(\Sigma_{b}, \Xi_{b}, \Omega_{b}\right.$ and $\left.\Sigma_{b}^{*}, \Xi_{b}^{*}, \Omega_{b}^{*}\right)$, the evolution equation turns out to be completely integrable. We find [11] that the following two operators,

$$
\mathbb{Q}_{1}=i\left(S_{+}^{(1)}+S_{+}^{(2)}\right), \quad \mathbb{Q}_{2}=S_{0}^{(1)} S_{+}^{(2)}-S_{0}^{(2)} S_{+}^{(1)} .
$$

commute with each other and with the evolution kernel $\mathbb{H}_{\uparrow \uparrow}$ so that they can be diagonalized simultaneously. The eigenfunctions are known in explicit form [23]:

$$
Q_{s, x}\left(z_{1}, z_{2}\right)=\frac{s}{z_{1}^{2} z_{2}^{2}} \int_{0}^{1} d \alpha\left(\frac{\alpha}{\bar{\alpha}}\right)^{i x} \exp \left[i s\left(\bar{\alpha} / z_{1}+\alpha / z_{2}\right)\right]
$$




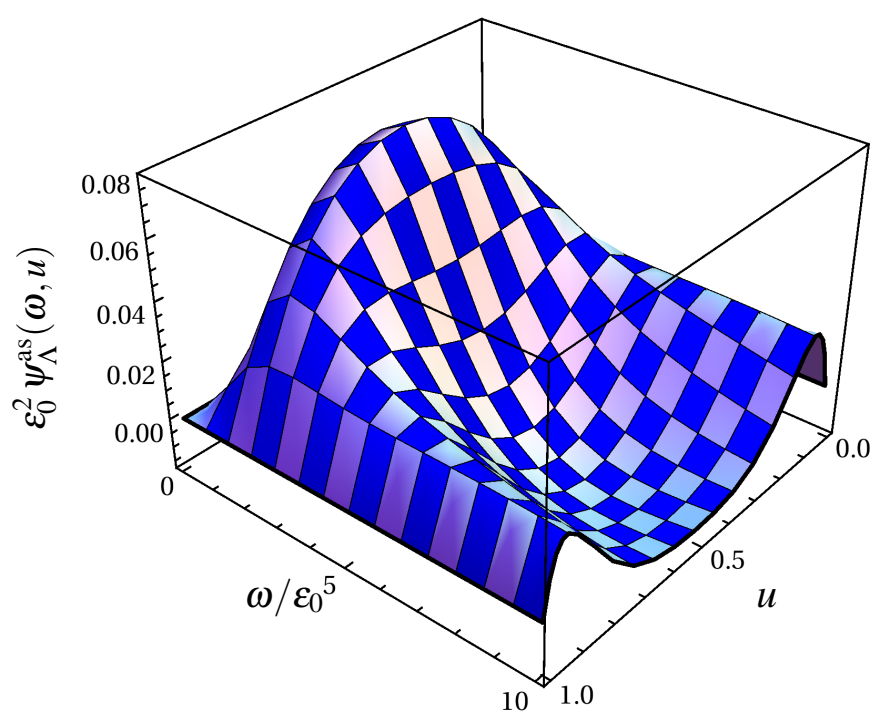

Figure 2: Asymptotic $\Lambda_{b}$ distribution amplitude for the simplest choice of the profile function $\xi_{0}\left(s, \mu_{0}\right)$.

where $s>0,-\infty<x, \infty$ and $\rho(x)=\pi x / \sinh (\pi x)$, and the corresponding anomalous dimensions form a continuum spectrum,

$$
\gamma(s, x ; \mu)=2 \ln \left(\mu s / s_{0}\right)+\mathscr{E}(x), \quad \mathscr{E}(x)=\psi(1+i x)+\psi(1-i x)+2 \gamma_{E}
$$

where $s_{0}=e^{2-\gamma_{E}}$. Going over to the momentum space and introducing new variables $\left\{\omega_{1}, \omega_{2}\right\} \rightarrow$ $\{\omega, u\}$ such that $\omega_{1}=u \omega, \omega_{2}=\bar{u} \omega$, the final result for the scale dependence of the baryon DA with aligned light quark helicities reads [11]

$$
\psi_{\perp}(\omega, u ; \mu)=\omega^{2} u \bar{u} \int_{-\infty}^{\infty} \frac{d x}{2 \pi} \int_{0}^{\infty} s d s \widetilde{Q}_{s, x}(\omega, u) L^{\frac{4}{3 \beta_{0}} \mathscr{E}(x)} R\left(s ; \mu, \mu_{0}\right) \eta_{\perp}\left(s, x ; \mu_{0}\right),
$$

with

$$
\widetilde{Q}_{s, x}(\omega, u)=\frac{1}{\omega} \sum_{n=0}^{\infty} \frac{4 i^{n}(2 n+3)}{(n+1)(n+2)} C_{n}^{3 / 2}(1-2 u) H_{n}(x) \frac{1}{\sqrt{s \omega}} J_{2 n+3}(2 \sqrt{s \omega}),
$$

where $\eta_{\perp}\left(s, x ; \mu_{0}\right)$ are nonperturbative functions defined at a low reference scale and $H_{n}(x)$ are Hahn polynomials in a suitable normalization, see [11].

The case with light quarks of opposite helicity, which is the only one relevant for the $\Lambda_{b}$ baryon, is more complicated. In this case integrability of the evolution equation is broken by the last term $-1 /\left(J_{12}\left(J_{12}-1\right)\right.$ in (4.5), which acts as a weak attractive interaction and creates a bound state - a discrete level in the spectrum of anomalous dimensions which is separated by the continuum by a finite gap [11]

$$
E_{0} \simeq-0.3214
$$

The value of the gap and the eigenfunction of the discrete level can be found, e.g., by the usual quantum-mechanical variational method. The corresponding solution can be viewed as an asymp- 
totic DA at large scales. We obtain

$$
\psi_{\Lambda}^{\mathrm{as}}(\omega, u ; \mu)=\omega^{2} u \bar{u} L^{\frac{4}{3 \beta_{0}} E_{0}} \int_{-\infty}^{\infty} \frac{d x}{2 \pi} \int_{0}^{\infty} s d s \widetilde{Q}_{s, x}(\omega, u) R\left(s ; \mu, \mu_{0}\right) \eta_{0}(x) \xi_{0}\left(s, \mu_{0}\right)
$$

where

$$
\eta_{0}(x) \simeq \frac{\sqrt{2} E_{0}}{\sqrt{2+x^{2}}} \frac{\rho(x)}{\left[E_{0}-\mathscr{E}(x)\right]}, \quad \quad \rho(x)=\pi x / \sinh (\pi x)
$$

is a good approximation. Note that this expression still contains an arbitrary nonperturbative function $\xi_{0}\left(s, \mu_{0}\right)$ which determines the distribution in overall momentum $\omega$ carried by the light quark pair. For the simplest ansatz

$$
\xi_{0}\left(s, \mu_{0}\right)=s e^{-s \varepsilon_{0}}
$$

we obtain (at the scale $\mu_{0}$ ) the DA shown in Fig. 2.

We expect that the model of the $\Lambda_{b}$ DA in Eq. (4.12) will be sufficient for phenomenological applications. A very similar model was suggested in Ref. [8] using different methods. Note that its shape is rather nontrivial: the deviation from the "naive" $\sim u \bar{u}$ behavior is increasing with the energy of the light quark pair, which might create a problem for factorization. This question requires a separate study.

Acknowledgments: This talk is based on the research reported in Refs. [9, 11, 12] and the author thanks S. Derkachov, A. Manashov and N. Offen for a very rewarding collaboration. Financial support by the DFG is gratefully acknowledged, grant BR2021/5-2.

\section{References}

[1] M. Beneke, G. Buchalla, M. Neubert and C. T. Sachrajda, $Q C D$ factorization for $B \rightarrow \pi \pi$ decays: Strong phases and CP violation in the heavy quark limit, Phys. Rev. Lett. 83 (1999) 1914 [hep-ph/9905312].

[2] M. Beneke, G. Buchalla, M. Neubert and C. T. Sachrajda, QCD factorization for exclusive, nonleptonic B meson decays: General arguments and the case of heavy light final states, Nucl. Phys. B 591 (2000) 313 [hep-ph/0006124].

[3] M. Beneke and T. Feldmann, Symmetry breaking corrections to heavy to light B meson form-factors at large recoil, Nucl. Phys. B 592 (2001) 3 [hep-ph/0008255].

[4] A. G. Grozin and M. Neubert, Asymptotics of heavy meson form-factors, Phys. Rev. D 55 (1997) 272 [hep-ph/9607366].

[5] B. O. Lange and M. Neubert, Renormalization group evolution of the B meson light cone distribution amplitude, Phys. Rev. Lett. 91 (2003) 102001 [hep-ph/0303082].

[6] V. M. Braun, D. Yu. Ivanov and G. P. Korchemsky, The B meson distribution amplitude in QCD Phys. Rev. D 69 (2004) 034014 [hep-ph/0309330].

[7] S. J. Lee and M. Neubert, Model-independent properties of the B-meson distribution amplitude, Phys. Rev. D 72 (2005) 094028 [hep-ph/0509350]. 
[8] G. Bell, T. Feldmann, Y.-M. Wang and M. W. Y. Yip, Light-Cone Distribution Amplitudes for Heavy-Quark Hadrons, JHEP 1311 (2013) 191 [arXiv:1308.6114 [hep-ph]].

[9] V. M. Braun and A. N. Manashov, Conformal symmetry of the Lange-Neubert evolution equation, Phys. Lett. B 731, 316 (2014) [arXiv:1402.5822 [hep-ph]].

[10] T. Feldmann, B. O. Lange and Y. M. Wang, B -meson light-cone distribution amplitude: Perturbative constraints and asymptotic behavior in dual space, Phys. Rev. D 89 (2014) 11, 114001 [arXiv:1404.1343 [hep-ph]].

[11] V. M. Braun, S. E. Derkachov and A. N. Manashov, Integrability of the evolution equations for heavy-light baryon distribution amplitudes, Phys. Lett. B 738, 334 (2014) [arXiv:1406.0664 [hep-ph]].

[12] V. M. Braun, A. N. Manashov and N. Offen, Evolution equation for the higher-twist B-meson distribution amplitude, Phys. Rev. D 92, no. 7, 074044 (2015) [arXiv:1507.03445 [hep-ph]].

[13] A. Khodjamirian, T. Mannel and N. Offen, Form-factors from light-cone sum rules with B-meson distribution amplitudes, Phys. Rev. D 75 (2007) 054013 [hep-ph/0611193].

[14] F. De Fazio, T. Feldmann and T. Hurth, SCET sum rules for $B \rightarrow P$ and $B \rightarrow V$ transition form factors, JHEP 0802 (2008) 031 [arXiv:0711.3999 [hep-ph]].

[15] V. M. Braun and A. Khodjamirian, Soft contribution to $B \rightarrow \gamma \ell v_{\ell}$ and the B-meson distribution amplitude, Phys. Lett. B 718, 1014 (2013) [arXiv:1210.4453 [hep-ph]].

[16] Y. M. Wang and Y. L. Shen, QCD corrections to $B \rightarrow \pi$ form factors from light-cone sum rules, Nucl. Phys. B 898, 563 (2015) [arXiv:1506.00667 [hep-ph]].

[17] M. Knödlseder and N. Offen, Renormalisation of heavy-light light ray operators, JHEP 1110 (2011) 069 [arXiv:1105.4569 [hep-ph]].

[18] H. Kawamura, J. Kodaira, C. F. Qiao and K. Tanaka, B meson light cone distribution amplitudes in the heavy quark limit, Phys. Lett. B 523 (2001) 111 [Erratum-ibid. B 536 (2002) 344] [hep-ph/0109181]

[19] A. P. Bukhvostov, G. V. Frolov, L. N. Lipatov and E. A. Kuraev, Evolution Equations for Quasi-Partonic Operators, Nucl. Phys. B 258 (1985) 601.

[20] S. E. Derkachov, G. P. Korchemsky and A. N. Manashov, Baxter Q operator and separation of variables for the open $S L(2, R)$ spin chain, JHEP 0310 (2003) 053 [hep-th/0309144].

[21] A. Ali, C. Hambrock, A. Y. Parkhomenko and W. Wang, Light-Cone Distribution Amplitudes of the Ground State Bottom Baryons in HQET, Eur. Phys. J. C 73, 2302 (2013) [arXiv:1212.3280].

[22] P. Ball, V. M. Braun and E. Gardi, Distribution Amplitudes of the $\Lambda_{b}$ Baryon in $Q C D$, Phys. Lett. B 665, 197 (2008) [arXiv:0804.2424 [hep-ph]].

[23] S. E. Derkachov, G. P. Korchemsky and A. N. Manashov, Separation of variables for the quantum SL(2,R) spin chain, JHEP 0307, 047 (2003) [hep-th/0210216]. 